\title{
ON A PROBLEM IN ERGODIC THEORY
}

\author{
BY V. S. VARADARAJAN ${ }^{1}$ \\ Communicated by Peter D. Lax, February 27, 1962
}

1. The present note is a summary of results obtained in the study of groups of automorphisms of a Borel space (the pertinent definitions are given in \$2). Our object is to generalize some aspects of the classical theory of von Neumann [15] and Kryloff-Bogoliouboff [11; 16], involving a single automorphism. The main results of this note are announced for countable but otherwise arbitrary groups of automorphisms of "sufficiently smooth" Borel spaces. Appropriate counterexamples can be given to illustrate the difficulties that arise when the "smoothness" restrictions on the Borel spaces are dropped. Details will be given in a future publication.

2. A Borel space is a pair $(X, B)$ where $X$ is an abstract set and $B$ a $\sigma$-algebra of subsets of $X$; when no confusion can arise we refer to $X$ itself as the Borel space. $(X, B)$ is countably generated if there is a denumerable sub-family $D \subseteq B$ such that $\beta$ is the smallest $\sigma$-algebra of subsets of $X$ containing $D .(X, B)$ is standard if the $\sigma$-algebra $B$ is $\sigma$-isomorphic to the $\sigma$-algebra of Borel subsets of a complete separable metric space. $(X, \beta)$ is analytic if $\beta$ is $\sigma$-isomorphic to the $\sigma$ algebra of Borel subsets of a separable metric space which is an analytic set in its completion. (For the relevant techniques involving standard and analytic spaces see $[2 ; 12 ; 14]$.) A measure $\nu$ on a Borel space $(X, B)$ is a nonnegative countably additive set function on $B$ with $\nu(X)=1$. If $\mathfrak{T}$ is a set of measures on $(X, B)$ we denote by $a(\mathfrak{T}, B)$ the smallest $\sigma$-algebra of subsets of $\mathfrak{T}$ with respect to which the maps $\tau_{A}: \nu \rightarrow \nu(A)$ are measurable for each $A \in \mathbb{B}$; when we speak of the Borel space $\mathfrak{T}$ we mean (T, $Q(\mathfrak{T}, B)$ ). An automorphism $\alpha$ of $(X, B)$ is a one-one map of $X$ onto itself such that $\alpha^{-1}(A) \in B$ if and only if $A \in B$. A $G$-space is a triple $(X, \Theta, G)$ where $(X, B)$ is a Borel space, $G$ a group and for each $g \in G$ there exists an automorphism $\alpha_{\theta}$ of $(X, B)$ such that $\alpha_{g_{1 g_{2}}}=\alpha_{g_{1}} \circ \alpha_{g_{2}}$ for all $g_{1}, g_{2} \in G$. The $G$-space is said to be countably generated (respectively standard, analytic) if $(X, B)$ is so. A measure $\nu$ on $(X, B)$ is $G$-invariant if $\nu(A)=\nu\left(\alpha_{g}^{-1}(A)\right)$ for all $A \in B$ and $g \in G$; it is $G$-ergodic if it is $G$-invariant and if $\nu(A)=0$ or 1 for any $A \in \mathbb{B}$ which is invariant, i.e., $\alpha_{g}^{-1}(A)=A$ for all $g \in G$. The

1 This work was done when the author was a temporary member of the Courant Institute of Mathematical Sciences during 1961-1962 under the partial support of the National Science Foundation. 
$\sigma$-algebra of all $A \in B$ which are invariant is denoted by $\bar{\Theta}_{G}$, and by $\bar{\AA}$ when no confusion can arise. We write $J_{X, B}$ (or $J$ ) and $\mathcal{E}_{X}, \mathbb{B}$ (or $\mathcal{E}$ ) for the set of $G$-invariant and $G$-ergodic measures respectively. It is well known, if $G$ is countable, that $\varepsilon$ is the set of extreme points of the convex set $\mathfrak{J}$. If $(X, \Theta, G)$ is a $G$-space we write $\mathfrak{I}=\{A: A \in \mathbb{B}, \mu(A)$ $=0$ for each $\mu \in \mathfrak{J}\}$ and $\overline{\mathfrak{N}}=\{A: A \in \mathfrak{N}, A$ invariant $\}=\mathfrak{N} \cap \bar{\beta}$. For any Borel space $(X, \beta)$ we write $\mathcal{F}$ for the Banach space of all real valued bounded functions $f$ on $X$ which are B-measurable with $\|f\|=\sup _{x \in X}|f(x)|$. If $X$ is a topological space we denote by $\mathfrak{C}(X)$ the space of bounded real valued continuous functions on $X$.

3. It is an important consequence of the von Neumann-KryloffBogoliouboff theory that every measure invariant under an automorphism of a sufficiently smooth Borel space is an integral of ergodic measures. Such representation theorems are, in special contexts, consequences of the general representation theorems of Choquet [4] and Bishop-deLeeuw [1]. For our theory we begin with the following much simpler proposition. This proposition can be immediately deduced from the Choquet-Bishop-deLeeuw theorems; but it is not necessary to do so since a simple proof of it is available. We remark also that in our special context we shall obtain representation theorems more general than those of Choquet and Bishop-deLeeuw (cf. Theorem 4).

THEOREM 1. Let $X$ be a normal Hausdorff space and $B$ the $\sigma$-algebra of Baire sets of $X$, i.e., the smallest $\sigma$-algebra of subsets of $X$ with respect to which all elements of $\mathrm{e}(X)$ are measurable. Let $\Gamma$ be a convex set of measures on $(X, B)$ which is compact in its weak*-topology and let $\Gamma^{\prime}$ be the set of extreme points of $\Gamma$. Then for a given $A \in B, \nu(A)=0$ for all $\nu \in \Gamma$ if and only if $\nu(A)=0$ for all $\nu \in \Gamma^{\prime}$.

The "only if" part is trivial. For the "if" part we may assume $A$ is a closed $G_{\delta}$. If we write $u=\sup \{\nu(A): \nu \in \Gamma\}$ and $\Gamma_{1}=\{\mu: \mu \in \Gamma$, $\mu(A)=u\}$ then it can be easily shown that $\Gamma_{1}$ is a nonempty closed (hence compact) convex subset of $\Gamma$. If $e$ is an extreme point of $\Gamma_{1}$ then $e \in \Gamma^{\prime}$ and this proves the theorem.

In the Kryloff-Bogoliouboff theory the notion of a time-average of a function plays an important role. Our basic observation is that if $\alpha$ is an automorphism of $(X, B), f$ a bounded $B$-measurable function and $\mu$ is any invariant measure, $\lim _{n \rightarrow \infty}(1 / n)(f(x)+f(\alpha x)+\cdots$ $\left.+f\left(\alpha^{n-1} x\right)\right)$ is the conditional expectation $E_{\mu}\left(f \mid \bar{B}_{\alpha}\right)$ of $f$ given the $\sigma$-algebra of sets in $B$ which are invariant under $\alpha$ (see $[6 ; 13]$ for a detailed discussion of conditional expectations and their properties). Our purpose now is to construct a mapping $U: f \rightarrow f^{*}$ with fairly 
reasonable properties such that for each $G$-invariant measure $\nu$, $f^{*}=E_{\nu}(f \mid \bar{B}) \nu$-almost everywhere.

Theorem 2. Let $(X, \beta, G)$ be a G-space, $G$ being a countable group. Then there exists a mapping $U$ of $\mathcal{F}$ into itself, $U: f \rightarrow f^{*}$ with the following properties: (i) If $f \geqq 0$, then $f^{*} \geqq 0$. (ii) $f^{*}$ is $\bar{B}$-measurable and $f^{*}=f$ if $f$ itself is $\bar{B}$-measurable. (iii) For any G-invariant measure $\nu, f^{*}$ $=E_{\nu}(f \mid \bar{\beta}) \nu$-almost everywhere. (iv) $\left\|f^{*}\right\| \leqq\|f\|$. Moreover if $U_{1}$ and $U_{2}$ are two such mappings and $f \in \mathcal{F}$, then $\left\{x:\left(U_{1} f\right)(x) \neq\left(U_{2} f\right)(x)\right\} \in \overline{\mathscr{N}}$.

By exhibiting $G$ as $\bigcup_{k=1}^{\infty} G_{k}$ where $G_{k}$ is generated by $k$ elements and $G_{1} \subseteq G_{2} \subseteq \cdots$, and utilizing the so-called martingale-reversed convergence theorem [13, pp. 389-396], we drop down to the construction of $U$ for the $G$-space $\left(X, \beta, G_{k}\right)$. For $k=1$ we are through using the Birkhoff ergodic theorem; the general case is settled by induction. If $G_{k+1}$ is the group generated by $G_{k}$ and a cyclic group $Z_{k+1}, V_{1}, V_{2}$ the mappings similar to $U$ for the $G$-spaces $\left(X, \Theta, G_{k}\right)$ and $\left(X, \Theta, Z_{k+1}\right)$ then we construct $U$ for $\left(X, ß, G_{k+1}\right)$ by utilizing the Hopf-ergodic theorem [10] or the recent results of Burkholder-Chow [3]; their application rests on the observation that $\bar{B} G_{k+1}=\bar{B} G_{k} \cap \bar{B} z_{k+1}$ where

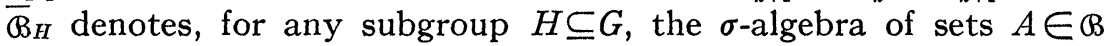
which are invariant under $H$.

Utilizing various technical devices we can now obtain the following theorem valid for standard $G$-spaces. A similar result can be proved for analytic $G$-spaces but its precise formulation is somewhat more delicate. We recall that $\mathcal{E}$ is the set of $G$-ergodic measures.

ThEOREM 3. Let $G$ be a countable group, let $(X, \beta, G)$ be a standard $G$-space and let $\Im \neq \varnothing$. Then $\mathcal{E} \neq \varnothing$ and there exists $a$ set $N \in \overline{\mathscr{T}}$ and $a$ map $\theta: x \rightarrow \theta_{x}$ of $X-N$ onto $\&$ with the following properties: (i) For each bounded B-measurable function $f$, the function $x \rightarrow \int_{X} f(y) d \theta_{x}(y)$ is $\bar{B}-$ measurable. In particular, $x \rightarrow \theta_{x}$ is a measurable map and $\theta_{\alpha_{g}(x)}=\theta_{x}$ for all $x \in X-N$ and $g \in G$. (ii) For any bounded B-measurable $f$ and any $G$-invariant measure $\nu$, we have $\int_{X} f(y) d \nu(y)=\int_{X-N}\left(\int_{X} f(y) d \theta_{x}(y)\right) d \nu(x)$. In particular, if we write $\tilde{f}$ for the function which is 0 over $N$ and is $\int_{X} f(y) d \theta_{x}(y)$ for any $x \in X-N$, then $\bar{f}=E_{\nu}(f \mid \bar{\beta}) \nu$-almost everywhere. If $x \rightarrow \theta_{x}^{\prime}$ is another such map, $\left\{x: \theta_{x}^{\prime} \neq \theta_{x}\right\} \in \bar{\Re}$, i.e., is an invariant set whose measure is zero under every invariant measure.

Corollary. For any $e \in \mathcal{E}$ write $E_{e}=\left\{x: x \in X-N, \theta_{x}=e\right\}$. Then $E_{e_{1}} \cap E_{e_{2}}=\varnothing$ if $e_{1} \neq e_{2}, \bigcup_{e \in \mathcal{E}} E_{e}=X-N$ and for $e^{\prime} \in \mathcal{E}, e^{\prime}\left(E_{\theta}\right)=1$ if $e^{\prime}=e,=0$ if $e^{\prime} \neq e$. Moreover, if $\nu$ is any invariant measure and $A \in \Theta$, we have 


$$
\nu(A)=\int_{X-N} \theta_{x}\left(A \cap E_{\theta x}\right) d \mu(x) .
$$

REMARK. It may be noted that the results of Theorem 3 have nonempty content as soon as $J \neq \varnothing$. We shall observe later that for any countable $G$ there are always standard $G$-spaces $(X, \beta, G)$ for which $J$ (and hence $\mathcal{E}$ ) are nonempty.

TheOREM 4. Let $G$ be a countable group, let $(X, B, G)$ be an analytic $G$-space, and let $J \neq \varnothing$. Then $\mathcal{E} \neq \varnothing$ and $\mathcal{E}$ is an analytic Borel space. Moreover, there exists an one-one correspondence $\sigma \rightarrow \nu$ between the set of all measures $\sigma$ on $\mathcal{E}$ and the set $J$ of all $G$-invariant measures $\nu$ on $(X, B)$ such that for all $A \in \mathbb{B}$

$$
\nu(A)=\int_{\mathcal{E}} e(A) d \sigma(e)
$$

Further if $(X, B, G)$ is a standard G-space then $\mathcal{E}$ is a standard Borel space.

When $X$ is a separable metric space and $B$ is the class of Borel subsets of $X$ we shall say that an $x \in X$ is regular if there exists an invariant set $E$ and an ergodic measure $e$ such that (i) $x \in E$, (ii) for any ergodic measure $e^{\prime}, e^{\prime}(E)=1$ or 0 according as $e^{\prime}=e$ or $e^{\prime} \neq e$, (iii) $e(W)>0$ for each open set $W$ containing $x$.

TheOREM 5. If $X$ is a separable metric space and $B$ is the class of Borel subsets of $X$ and if $(X, B, G)$ is standard, then we can choose $N_{1} \in \overline{\mathscr{X}}$ such that each $x \in X-N_{1}$ is regular.

One can exhibit a wide class of situations in which our theory has content. Let $Y$ be a compact metric space and $\mathfrak{e}$ the class of Borel subsets of $Y$. Let $G$ be a countable group. We write $Y_{G}$ for the space of all functions $y$ from $G$ to $Y$ and $\mathfrak{e}_{G}$ for the smallest $\sigma$-algebra of subsets of $Y_{G}$ with respect to which the maps $y \rightarrow y(g)$ are measurable for each $g \in G$. Under the product topology $Y_{G}$ is a compact metric space and $\mathcal{C}_{G}$ is the class of Borel subsets of $Y_{G}$. For any $g \in G$ and $y \in Y_{G}$ we define $\alpha_{g}(y) \in Y_{G}$ by $\alpha_{g}(y)(h)=y(h g)$. Then $\alpha_{g}$ is a homeomorphism of $Y_{G}$ and $\left(Y_{G}, \mathfrak{e}_{G}, G\right)$ is a standard $G$-space. It can be easily shown that $\mathcal{J}$, and a fortiori $\mathcal{E}$, are nonempty. It may also be remarked that if $X$ is any compact metric space and $G$ a suitably restricted countable group of homeomorphisms of $X$, one can assert the existence of invariant measures [7].

As for counterexamples we have

THEOREM 6. For any infinite countable $G$ there are countably gener- 
ated $G$-spaces $(X, \Theta, G)$ such that there exist continuum many $G$-invariant measures but no G-ergodic measures.

Remarks. (1) If $X$ is a compact metric space and $G$ is a countable group of homeomorphisms of $X$, the representation given in Theorem 4 is an immediate consequence of the theorem of Choquet [4]. It may be remarked that in this case one need not even assume that $G$ is countable. But when $G$ is uncountable, an example due to Kolmogoroff [7] indicates that the decomposition theory outlined in Theorem 3 and its corollary may not obtain; even the weaker Theorem 7 may fail to be valid.

(2) Let $(Y, \mathfrak{C})$ be a Borel space and $(X, \Re)$ the cartesian product of countably many copies of $(Y, \mathcal{e})$. We regard $X$ as the space of functions $x$ from $\{1,2, \cdots\}$ into $Y$ and for any permutation $g$ of the integers $\{1,2, \cdots\}$ we define $\alpha_{g}: x \rightarrow x^{\prime}$ by setting $x^{\prime}(n)=x\left(g^{-1}(n)\right)$ for $n=1,2, \cdots$. When $(Y, \mathfrak{e})$ is analytic and $G$ is the group of all $g$ which move only finitely many integers, Theorem 4 can be applied to $(X, B, G)$ and contains essentially the results of De Finetti [5] and Hewitt-Savage [9].

4. It may not be without interest to make a few remarks on a way of reformulating the problem of representing invariant measures as integrals of ergodic measures which applies to all Borel spaces. Let $G$ be countable and $(X, \Theta, G)$ a $G$-space. A $\sigma$-algebra $D \subseteq \Theta$ is said to be of type $(R)$ if (i) Every set in $D$ is invariant i.e. $D \subseteq \bar{B}$, (ii) if $A \in B$ there exists a set $B \in \mathbb{D}$ such that $A \triangle B=(A-B) \cup(B-A) \in \overline{\mathfrak{T}}$, (iii) if $\nu_{1}$ and $\nu_{2}$ are two invariant measures such that $\nu_{1}(A)=\nu_{2}(A)$ for all $A \in \mathbb{D}$, then $\nu_{1}=\nu_{2}$. Obviously if there exists a $D$ of type $(R)$ then $\bar{B}$ is itself of type $(R)$.

TheOREM 7. If $G$ is countable, then for any Borel space $(X, B, G)$, $B$ is of type $(R)$. If $(X, B)$ is countably generated (respectively standard, analytic) there exist countably generated (respectively standard, analytic) $\sigma$-algebras of type $(R)$.

We shall say that a $G$-space $(X, \Theta, G)$ has sufficiently many ergodic measures if for any invariant set $A \in B, \nu(A)=0$ for all invariant measures $\nu$ if and only if $e(A)=0$ for all ergodic $e$. Clearly any analytic $G$-space has sufficiently many ergodic measures.

Theorem 8. Let $G$ be a countable group and $(X, \Theta, G)$ a G-space. Then given any measure $\sigma$ on $(X, \bar{B})$ such that $\sigma(N)=0$ for all $N \in \bar{\Re}$ there exists a unique invariant measure $\nu$ on $(X, B)$ such that $\nu(A)$ $=\sigma(A)$ for all $A \in \bar{B}$. Moreover, in order that every invariant measure 
be expressible as a unique integral of ergodic measures it is necessary and sufficient that $(X, \Theta, G)$ have sufficiently many ergodic measures.

The writer wishes to thank Dr. R. Ranga Rao, Dr. R. Sacksteder and Dr. H. Teicher for several interesting discussions on the circle of ideas around which this note is centered.

Added in Proof. Since this note was submitted the author has extended the present results to the case of Borel $G$-spaces, $G$ a separable locally compact group; details will be given in the publication mentioned at the beginning.

\section{REFERENCES}

1. E. Bishop and $\mathrm{K}$. de Leeuw, The representations of linear functionals by measures on sets of extreme points, Ann. Inst. Fourier Grenoble 9 (1959), 305-331.

2. D. Blackwell, On a class of probability spaces, Proc. 3rd Berkeley Sympos. Mathematical Statistics and Probability, 1954-1955, Vol. II, pp. 1-6, University of California Press, Berkeley and Los Angeles, 1956.

3. D. L. Burkholder and Y.S. Chow, Iterates of conditional expectation operators, Proc. Amer. Math. Soc. 12 (1961), 490-495.

4. G. Choquet, Existence et unicitê des représentations intégrales, Séminaire Bourbaki, Décembre 1956, 139-01 to 139-15.

5. B. De Finetti, La prévision, ses lois logiques, ses sources subjectives, Ann. Inst. Henri Poincaré 7 (1937), 1-68.

6. J. L. Doob, Stochastic processes, Wiley, New York, 1953.

7. S. V. Fomin, On measures invariant under certain groups of transformations, Izv. Akad. Nauk. SSSR 14 (1950), 261-274. (Russian)

8. P. R. Halmos, Measure theory, Van Nostrand, New York, 1950.

9. E. Hewitt and L. J. Savage, Symmetric measures on cartesian products, Trans. Amer. Math. Soc. 80 (1955), 470-501.

10. E. Hopf, The general temporally discrete Markoff process, J. Rational Mech. Anal. 3 (1954), 13-45.

11. N. Kryloff and N. Bogoliouboff, La thêorie gênérale de la mesure dans son application a l'étude des systemes dynamiques de la mécanique non linéaire, Ann. of Math. 38 (1937), 65-113.

12. C. Kuratowski, Topologie. I, Warsaw, 1948.

13. M. Loève, Probability theory, Van Nostrand, New York, 1955.

14. G. W. Mackey, Borel structures in groups and their duals, Trans. Amer. Math. Soc. 85 (1957), 134-165.

15. J. von Neumann, Zur Operatorenmethode in der klassischen Mechanik, Math. Ann. 33 (1932), 587-642.

16. J. C. Oxtoby, Ergodic sets, Bull. Amer. Math. Soc. 58 (1952), 116-136.

Institute of Mathematical Sciences, New York University 